\title{
2 Pharmakologie/Narkoseführung
}

\author{
Martin Zoremba
}

\subsection{Grundlagen}

Im Rahmen der Adipositas ist wegen der modifizierten Medikamentenverteilung in unterschiedlichen Geweben mit einer veränderten Pharmakokinetik bzw. einem veränderten Verteilungsvolumen zu rechnen. Die Adipositas führt zu einer größeren fettfreien Körpermasse (Lean Body Mass) und zu einem höheren Fettanteil als bei schlanken Individuen gleicher Größe, gleichen Alters und Geschlechts. Die fettfreie Masse repräsentiert 20-40\% der Körpermasse. Bei normal schlanken Individuen beträgt der Blutfluss zum Fett nur etwa 5\%, wohingegen er bei adipösen Patienten deutlich ansteigt.

Über die Effekte der Adipositas auf die Plasmaproteine besteht keine Einigkeit. Die erhöhten Konzentrationen von Triglyzeriden, Cholesterol, Lipoproteinen und freien Fettsäuren können die freien Plasmakonzentrationen von Medikamenten ansteigen lassen. Zudem kann die Leberfunktion durch die Fettinfiltration eingeschränkt sein. Studien belegen, dass Adipositas auch die Nierenfunktion verschlechtert und einen erhöhten Risikofaktor für die terminale Niereninsuffizienz darstellt. In der Summe führt dies zu einer schlechteren Steuerbarkeit der Prämedikations/Narkosewirkung und erhöht die Neben/Wechselwirkungen der Narkosemedikamente. Diese kommen in der unmittelbaren postoperativen Phase zum Tragen und können die nach Allgemeinanästhesie kompromittierte respiratorische Funk- tion negativ beeinflussen. Zusätzliche Faktoren, insbesondere ein erhöhtes Patientenalter, üben einen ausgeprägten additiven Effekt aus.

Daher wird für die meisten Pharmaka die Dosisberechnung anhand des idealen Körpergewichtes durchgeführt.

Idealerweise sind Medikamente mit stabiler Pharmakokinetik zu bevorzugen bzw. multimodale Konzepte anzuwenden, welche auch den Einsatz von Regionalanästhesieverfahren sowie ggf. laparoskopische Operationstechniken beinhalten und individuell auf den Patienten zugeschnitten sind. Ziel muss es sein, eine gute Steuerbarkeit der Narkose mit maximal möglicher Stressreduktion ohne größere hämodynamische Nebenwirkungen zu erreichen. Nachwirkende pharmakologische Effekte (z.B. postoperative Restcurarisierung, PORC) sollten bei der Auswahl der Narkose/Anästhesieverfahren beachtet werden, um eine rasche Erholung der kognitiven aber auch der Organfunktionen zu ermöglichen.

Narkosekonzepte im Sinne einer "Fast-Track-Anästhesie" sollten angewandt werden 


\subsection{Muskelrelaxantien}

Im Rahmen einer Allgemeinanästhesie werden Muskelrelaxantien zur Intubation sowie für die intraoperative Optimierung des Operationssitus regelmäßig benutzt. Der Einsatz von Muskelrelaxantien (Intubationsnarkose) verursacht eine Kranialverlagerung des Zwerchfells (Tonusverlust), welche die Entstehung von Kompressionsatelektasen begünstigt. Darüber hinaus kann eine initiale bzw. repetitive Relaxierung zu einer Postoperative Residual Curarisation (PORC) führen, die sich negativ auf die pulmonale Funktion auswirkt und die Morbidität signifikant erhöht. Ein konsequentes quantitatives neuromuskuläres Monitoring kann nachweislich die Inzidenz an postoperativ messbaren Restrelaxierungen deutlich reduzieren. Konstatiert werden muss jedoch, dass diese Methode eine ausgeprägte Messungenauigkeit aufweist, was dazu führt, dass bei einem nicht unerheblichen Anteil der Patienten mit klinisch bedeutsamer Restrelaxierung zu rechnen ist. Die Variabilität der Wirkdauer ist sowohl bei einmaliger als auch bei repetitiver Gabe von nichtdepolarisierenden Muskelrelaxantien direkt abhängig vom Medikament. Neben der individuell unterschiedlichen Medikamentenelimination über die Organe (Leber/Niere), kommt es mit steigendem Patientenalter zu einer größeren Variabilität der Wirkdauer. Benzylisochinoline, welche einer geringeren organabhängigen Elimination unterliegen, sind diesbezüglich zu bevorzugen. Daher sollte der Einsatz von Muskelrelaxantien nur nach Bedarf erfolgen bzw. Alternativverfahren (Larynxmaske, Regionalanästhesie) in Erwägung gezogen werden. Zusätzlich sollte die Medikamentendosierung entsprechend angepasst werden.

\subsubsection{Benzylisochinolinderivate}

\section{Cisatracurium}

Cisatracurium wird weitestgehend organunabhängig eliminiert. Es besitzt nachweislich die geringste Variabilität der Wirkdauer und sollte bei Risikopatienten (Adipositas, Geriatrie, Niereninsuffizienz) vorrangig eingesetzt werden. Es besitzt das geringste anaphylaktische Potenzial aller aktuellen Muskel-

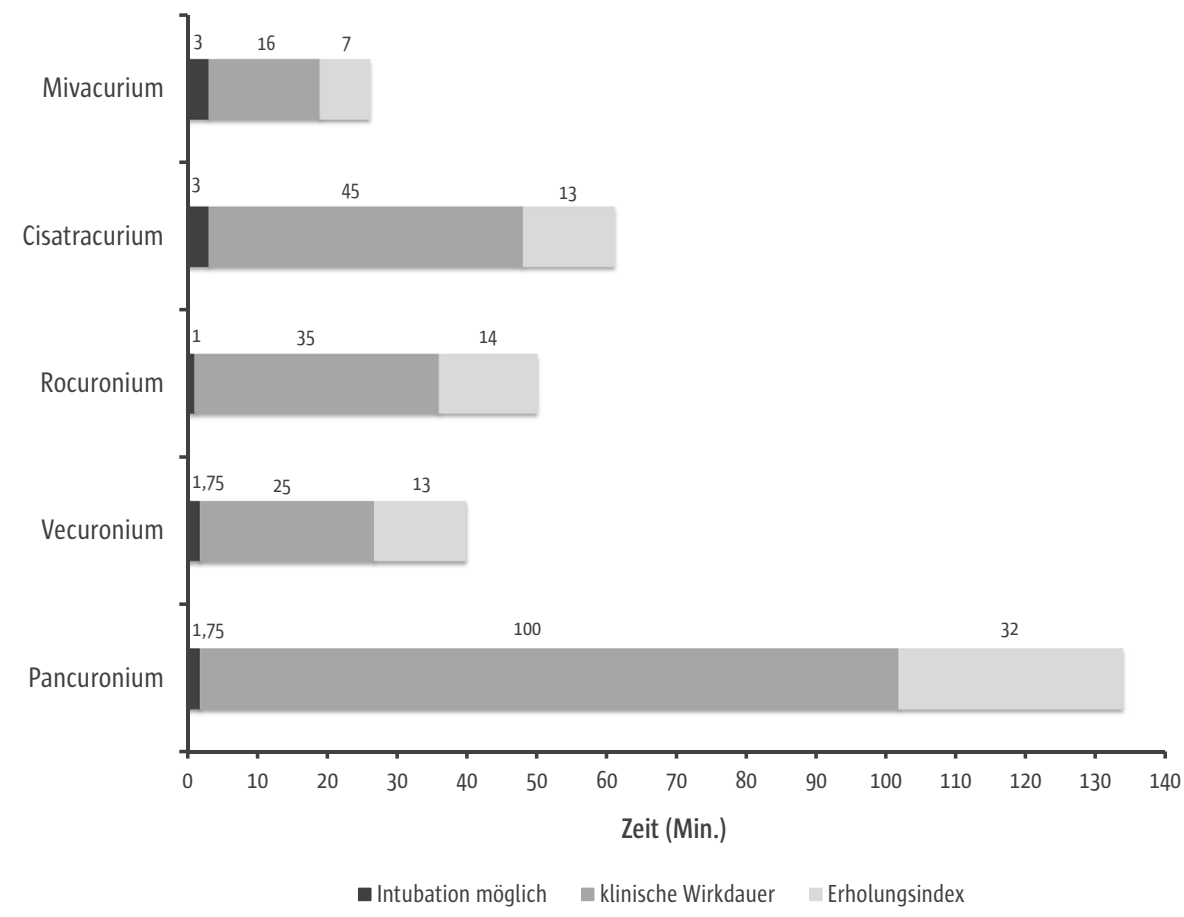

Abb. 1 Pharmakologische Kenndaten der nichtdepolarisierenden Muskelrelaxantien 
relaxantien. Nachteilig ist die etwas verzögerte Anschlagzeit (s. Abb. 1).

\section{Mivacurium}

Mivacurium wird durch im Plasma vorhandene Pseudocholinesterasen abgebaut. Es besitzt die kürzeste Wirkdauer aller aktuellen nicht depolarisierenden Muskelrelaxantien (NDMR). Das anaphylaktische Potenzial bei rascher Injektion sollte beachtet werden. Die Anschlagzeit ist vergleichbar der von Cisatracurium.

Bei Vorhandensein von atypischen Pseudocholinesterasen ist die Wirkdauer (v.a. bei homozygoten Genträgern) extrem verlängert. Bei Verdacht auf atypische Pseudocholinesterase sollte die „Dibucainzahl“ laborchemisch bestimmt werden.

\subsubsection{Steroidderivate}

\section{Rocuronium}

Rocuronium wird primär hepatisch $(\sim 80 \%)$ und teilweise renal eliminiert. Es besitzt die schnellste Anschlagszeit aller nicht depolarisierenden Muskelrelaxantien (NDMR). Eine rapid sequence induction (RSI) ist mit der 3 x ED95 möglich, d.h., mit dem 3-fachen der Dosis, um eine 95\%-ige neuromuskuläre Erregungsübertragung zu blockieren. Allerdings ist dadurch eine deutlich verlängerte Wirkung der neuromuskulären Blockade zu beachten. Aufgrund der Metabolisierung kann auch nach einmaliger Applikation eine Wirkungsverlängerung von über 50\% auftreten.

\subsubsection{Depolarisierende Muskelrelaxantien}

\section{Succinylcholin}

Succinylcholin wird durch die Pseudocholinesterase abgebaut und besitzt die kürzeste Wirkdauer aller klinisch relevanten Muskelrelaxantien. Die Wirkdauer kann bei Vorhandensein von atypischen Cholinesterasen verlängert sein (s. Kap. XII.2.2.1). Aufgrund der zahlreichen Nebenwirkungen (Herzrhythmusstörungen, Hyperkaliämie, Faszikulationen, maligne Hyperthermie) sollte auf Succinylcholin im Rahmen von Elektiveingriffen verzichtet werden.

\subsection{Opioide}

Perioperativ verabreichte Opioide besitzen bei adipösen Patienten ein verändertes Wirkprofil (s. Tab. 2). Die substanzspezifische Lipidlöslichkeit und die damit verbundene Akkumulation im Fettgewebe sind von Bedeutung. Darüber hinaus ist mit einem erhöhten Verteilungsvolumen sowie einer veränderten Medikamentenclearance zu rechnen, wodurch sich die pharmakologisch wirksamen Plasmaspiegel nur schwer abschätzen lassen. Während für die mittellang bis lang wirksamen Opioide Fentanyl und Sufentanil diese Einschränkung zutreffend ist, zeigt das kurzwirksame Opioid Remifentanil keine nennenswerte Veränderung der Pharmakokinetik bzw. des Verteilungsvolumens bei Adipositas. Die analgetische Wirkdauer ist jedoch deutlich verkürzt, was sich negativ auf die postoperative Schmerztherapie auswirkt. Aus diesem Grund ist eine Kombination mit einer reduzierten Dosis eines langwirksamen Opioides vorteilhaft. Neben den direkt über die Opioidrezeptoren vermittelten Effekten, spielt bei Adipositas die Interaktion zwischen Hypnotika und Muskelrelaxantien eine besondere Rolle. Hierbei kommt es $\mathrm{zu}$ additiven Effekten sowohl hinsichtlich der Atemregulation als auch bezüglich der perioperativen Muskelkraft und Vigilanz. Zusammenfassend begünstigt dies einen „upper Airway“ Kollaps, was insbesondere in der unmittelbaren postoperativen Phase zu vermehrter Desaturation mit Gefahr der Hypoxämie führen kann.

Initial ist eine ausreichend hohe (loading) Dosis eines langwirksamen Analgetikums (Fentanyl/Sufentanil) gefolgt von einer Remifentanilinfusion für Schmerzspitzen zu verabreichen. Repetitionsdosen der langwirksamen Analgetika sollten deutlich niedriger dosiert werden als die „loading"-Dosis. Eine Kombination mit einem Regionalanästhesieverfahren ist zu prüfen!

\subsection{Hypnotika}

Analog zu den Opioiden besitzen auch Hypnotika eine im Vergleich zu normalgewichtigen Patienten veränderte Pharmakokinetik (s. Tab. 2). Während Propofol wegen der kurzen Eliminationshalbwertzeit als das Einleitungshypnotikum der Wahl angesehen wird, ist aufgrund der substanzspezifischen Lipophilie bei kontinuierlicher prolongierter Infusion mit einer Akkumulation zu rechnen. 
Tab. 2 Dosierungsempfehlungen für die gebräuchlichsten Anästhetika (mod. nach Meißner 2008, Casati A. et al. 2004, mit freundlicher Genehmigung der Georg Thieme Verlag KG)

\begin{tabular}{|c|c|c|c|c|c|c|c|c|}
\hline \multirow[b]{2}{*}{ Substanz } & \multicolumn{2}{|c|}{$\begin{array}{l}\text { Verteilungsvolumen } \\
\text { (I) }\end{array}$} & \multicolumn{2}{|c|}{$\begin{array}{l}\text { Clearance } \\
\left(\mathrm{ml} \times \min ^{-1}\right)\end{array}$} & \multicolumn{2}{|c|}{$\begin{array}{l}\text { terminale Eliminations- } \\
\text { halbwertzeit (h) }\end{array}$} & \multicolumn{2}{|c|}{ Dosierungsempfehlungen } \\
\hline & normal & adipös & normal & adipös & normal & adipös & Induktion & $\begin{array}{l}\text { kontinuierliche } \\
\text { Infusion }\end{array}$ \\
\hline Thiopental & - & - & 197,2 & 416,3 & 6,3 & 27,8 & $\downarrow$ & nicht empfohlen \\
\hline Propofol & 13 & 17,9 & 28,3 & 24,3 & 4,1 & 4,0 & TKW & TKW \\
\hline Midazolam & 114 & 311 & 530 & 472 & 2,3 & 5,9 & TKW & IKW \\
\hline Rocuronium & 14,6 & 11,8 & 0,45 & 0,03 & 1,1 & 1,2 & IKW & $\downarrow$ \\
\hline Cis-Atracurium & 8,5 & 8,6 & 440 & 444 & 0,2 & 0,2 & IKW & $\downarrow$ \\
\hline Sufentanil & 346 & 547 & 1780 & 1990 & 2,1 & 2,1 & TKW & $\downarrow$ \\
\hline Fentanyl & n.d. & n.d. & 718 & 986 & n.d. & n.d. & \multicolumn{2}{|c|}{$\begin{array}{c}\mathrm{PK}=52 \times 1 / \text { Korrektur-Faktor } \\
(\sim 2>140 \mathrm{~kg})\end{array}$} \\
\hline Remifentanil & 6,8 & 7,5 & 2700 & 3100 & & & IKW & IKW \\
\hline Succinylcholin & n.d. & n.d. & n.d. & n.d & & & TKW & nicht empfohlen \\
\hline
\end{tabular}

IKW = ideales Körpergewicht; $n . d$ = nicht ermittelt aufgrund eines nicht linearen Zusammenhangs; PK = Pharmakokinetische Masse;

TKW = totales Körpergewicht; $\downarrow=$ reduzierte Dosis

Der Einsatz von Inhalationsanästhetika bei adipösen Patienten im Sinne einer „balancierten Anästhesie“ gegenüber einer „TIVA“ wird kontrovers diskutiert. Bei Einsatz von volatilen Anästhetika ist eine genaue Abwägung der Nutzen/Risikorelation der potenziell wichtigen Nebenwirkungen (maligne Hyperthermie/postoperative nausea and vomiting, PONV) durchzuführen.

Volatile Anästhetika bieten theoretisch besonders bei adipösen Patienten einige Vorzüge. Die Substanzen Sevofluran und Desfluran werden nur zu einem geringen Teil metabolisiert, akkumulieren nicht bzw. gering im Fettgewebe und sind aufgrund ihrer geringen Löslichkeit im Blut gut steuerbar. Außerdem bietet der endexspiratorische MAC-Wert (minimum alveolar concentration, minimale alveoläre Konzentration) eine gute Möglichkeit, die Narkosetiefe abzuschätzen. Hierbei zeigt Desfluran die stabilsten pharmakokinetischen Eigenschaften aller volatilen Anästhetika. Im Gegensatz zu Sevofluran ist selbst bei Adipositas nicht mit einer veränderten $\mathrm{Ki}$ netik zu rechnen. Neuere Untersuchungen konnten zeigen, dass eine balancierte Anästhesietechnik gegenüber einer total intravenösen Anästhesie mit Propofol hinsichtlich verbesserter Fast-track-Scores und Lungenfunktion der unmittelbaren postoperativen Phase einhergeht.

\subsubsection{Propofol}

Propofol ist das am meisten verwendete intravenöse Hypnotikum. Charakteristisch sind eine ausgeprägte Lipophilie sowie der schnelle Wirkungseintritt aufgrund einer raschen Penetration der Blut-HirnSchranke gefolgt von einer Umverteilung in Muskelund Fettgewebe. Zur Dosisberechnung wird daher das totale Körpergewicht gewählt. Obwohl es, v.a. bei prolongierter Applikation, zu einer Akkumulation im Fettgewebe kommt, unterscheidet sich die terminale Eliminationshalbwertzeit nicht von der eines normalgewichtigen Kollektivs. Dadurch besitzt es hervorragende Eigenschaften zur Durchführung einer totalen intravenösen Anästhesie (TIVA). Ungeachtet dessen ist gegenüber einer balancierten Anästhesie bzw. dem volatilen Anästhetikum Desfluran mit einem verzögerten Aufwachverhalten zu rechnen. Eine konsequente Überwachung der Narkosetiefe $\left(\right.$ BIS $^{\circledast}$, Narko-trend $\left.{ }^{\circledast}\right)$ sowie der Einsatz von „target controlled infusion“ (TCI) Systemen kann die Ausleitungzeit verbessern sowie den Propofolverbrauch signifikant reduzieren.

Eine Dosisreduktion von Propofol durch Einsatz von Opioiden (Remifentanil) sowie einer "target controlled infusion" sollte angestrebt werden. 


\subsubsection{Benzodiazepine (Midazolam)}

Benzodiazepine sind stark lipophile Substanzen. Dies bedingt im Rahmen der Adipositas eine ausgeprägte Verteilung im Fettgewebe sowie eine verlängerte Elimination. Insgesamt ist die Wirkung, insbesondere bezüglich respiratorischer Effekte, nur schwer vorherzusagen, was durch zusätzliche Komorbiditäten negativ beeinflusst werden kann (OSA, Alter, Herzinsuffizienz). Selbst geringe Mengen von Benzodiazepinen können eine zentrale Atemstörung sowie durch den muskelrelaxierenden Effekt einen „upper airway collapse " hervorrufen und damit kritische Desaturationen begünstigen. Daher sollte generell auf den Einsatz von Benzodiazepinen verzichtet und auf besser steuerbare Substanzen ausgewichen werden.

Benzodiazepine sind wegen der Gefahr der "upper airway collaps"selbst bei niedriger Dosierung zu vermeiden!

\subsubsection{Inhalationsanästhetika}

Generell sind alle volatilen Anästhetika lipophile Substanzen. Die Akkumulation der Inhalationsanästhetika ist der wichtigste Faktor für die Dauer der Narkoseausleitung. Allerdings gibt es große Unterschiede hinsichtlich der Eliminationgeschwindigkeit. Diesbezüglich weist Desfluran die besten Eigenschaften auf. Während Desfluran eine im Vergleich zu normalgewichtigen Patienten nahezu identische Eliminationsgeschwindigkeit bei Adipositas besitzt, ist diese bei Sevofluran deutlich verlängert. Dennoch sollten hohe Konzentrationen von Desfluran aufgrund der dabei vermehrt auftretenden bronchialen Irritationen vermieden werden. Dies zeigt sich in verlängerter Ausleitungszeit sowie einer verzögerten postoperativen kognitiven Funktion sowohl bei Verwendung von Desfluran als auch von Propofol. Eine Dosisreduktion durch Einsatz von Remifentanil und/oder einem Regionalanästhesieverfahren bzw. die exakte Dosierung (Erfassung der Narkosetiefe) könnten diese pharmakologischen Eigenschaften nivellieren.

Aufgrund der verlängerten Auswaschphase von Sevofluran gegenüber Desfluran bei Adipositas sollte durch ein zusätzliches Regionalanästhesieverfahren und/oder dem additiven Einsatz von Remifentanil eine Dosisreduktion angestrebt werden.

\section{Literatur}

Casati A, Marchetti C, Spreafico E, Mamo D (2004) Effects of obesity on wash-in and washout kinetics of sevoflurane. Eur | Anaesthesiol 21, 243-245

Cheymol G (2000) Effects of obesity on pharmacokinetics implications for drug therapy. Clin Pharmacokinet 39, 215-231

Diefenbach C (2005) Muskelrelaxantien und ihre Überwachung. Anaesth Intensivmed 46, 233-246

Eastwood PR, Platt PR, Shepherd K, Maddison K, Hillman DR (2005) Collapsibility of the upper airway at different concentrations of propofol anesthesia. Anesthesiology 103, 470-477

Juvin P, Vadam C, Malek L, Dupont H, Marmuse IP, Desmonts JM (2000) Postoperative recovery after desflurane, propofol, or isoflurane anesthesia among morbidly obese patients: a prospective, randomized study. Anesth Analg 91, 714-719

Meißner A (2008) Anästhesie bei Patienten mit Adipositas. Besonderheiten des perioperativen Managements. Anästhesiol Intensivmed Notfallmed Schmerzther 43, 270-277

Shibutani K, Inchiosa MA Jr, Sawada K, Bairamian M (2004) Accuracy of pharmacokinetic models for predicting plasma fentanyl concentrations in lean and obese surgical patients: derivation of dosing weight (,pharmacokinetic mass'). Anesthesiology $101,603-613$

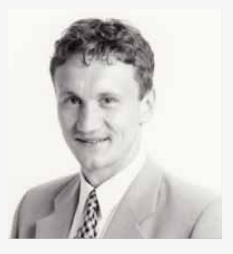

PD Dr. med. Martin Zoremba, D.E.A.A.

Nach Beendigung des Medizinstudiums an der Philipps Universität Marburg absolvierte er sein AIP an der Universität Gießen und ist seit 2000 in der Abteilung für Anästhesie und Intensivtherapie an der Universität Marburg tätig. Sein Forschungsschwerpunkt ist das perioperative Management von adipösen Patienten. Ein wichtiger Aspekt ist die Evaluation von „Fast-tracking-Prozeduren“ für Risikopatienten mit besonderem Fokus auf die perioperative respiratorische Funktion sowie postoperative schlafbezogene Atemstörungen. 